\title{
SUPERPOSABILITY PROPERTIES OF NATURALLY METRIZED GROUPS ${ }^{1}$
}

\author{
DAVID ELLIS
}

Let $p$ and $q$ be elements of an additively written abelian group $G$. The unordered pair of elements $(p-q, q-p)$ is written $p q=|p-q|$ and is called the "distance" of $p$ and $q$. This distance has the properties of symmetry $(p q=q p)$ and vanishing $(p q=|0|$ if and only if $p=q$ ). The group bearing this distance is called naturally metrized.

Two subsets of $G$ are congruent provided there is a distance-preserving mapping of one onto the other. A congruence of $G$ with itself is a motion of $G$. Two subsets of $G$ are superposable if one is mapped onto the other by a motion of $G$. $G$ has the property of free mobility if any congruence between any two subsets of $G$ can be induced by a motion of $G .^{2}$

It was shown by Menger ${ }^{3}$ that any two congruent pairs of elements of $G$ are superposable. In this note it is shown that $G$ has the property of free mobility.

If $t \in G$ the mapping $x \rightarrow x \oplus t$ of $G$ onto itself is the translation induced by $t$. The reflection in $O$ of $G$ is the mapping $x \rightarrow-x$ of $G$ onto itself. Clearly all translations and the reflection in $O$ are congruences. Denote by $\Gamma$ the group of motions of $G$ obtained by adjoining the reflection in $O$ to the group of all translations of $G$.

Theorem. Let $S \subset G$ and $\alpha$ be a mapping of $S$ onto $T \subset G$. A necessary and sufficient condition that there be a motion $\mu \in \Gamma$ which induces $\alpha$ over $S$ is that $a b=a \alpha b \alpha$ for all $a, b \in G$, that is, $\alpha$ is a congruence.

Proof. ${ }^{4}$ The necessity is obvious. Suppose that $\alpha$ is a congruence.

Presented to the Society, June 19, 1948; received by the editors April 9, 1948, and, in revised form, May 14, 1948.

${ }^{1}$ This paper is part of a dissertation being written for the $\mathrm{Ph} . \mathrm{D}$. degree, University of Missouri, under the direction of L. M. Blumenthal.

2 The concepts of superposability and free mobility vis-di-vis congruence have recently assumed new importance in certain of the characterization problems of metric geometry (cf. Garrett Birkhoff, Metric foundations of geometry. I, Trans. Amer. Math. Soc.vol. 55 (1944) pp. 465-492). These notions, logically equivalent in euclidean, spherical, and hyperbolic spaces, are quite distinct, for example, in elliptic space where they have been extensively studied in a recent publication (cf. L. M. Blumenthal, Congruence and superposability in elliptic space, Trans. Amer. Math. Soc. vol. 62 (1947) pp. 431-451).

${ }^{3} \mathrm{~K}$. Menger, Beiträge zur Gruppentheorie I. Über einer Abstand im Gruppen, Math. Zeit. vol. 33 (1931) pp. 396-418.

${ }^{4}$ The author is indebted to the referee for this more concise form of the original proof. 
Since $\Gamma$ contains all translations and the group of translations is transitive over $G$, we may assume without loss of generality that $0 \in S$ and that $0 \alpha=0$. Denote by $S^{\prime}$ the subset of $S$ which is invariant under $\alpha$. We distinguish two cases:

Case 1. $x \in S^{\prime}$ implies $2 x=0$.

Suppose $w \in S, w \in S^{\prime}$. Then $w 0=w \alpha 0 \alpha=w \alpha 0$. Since $w \notin S^{\prime}, w \neq w \alpha$. However, $(w,-w)=(w \alpha,-w \alpha)$ so that $w \alpha=-w$. Apply the reflection in $O$ over $G . S^{\prime}$ is invariant under the reflection and for all other elements of $S,-w=w \alpha$ so that the reflection coincides with $\alpha$ over $S$.

Case 2. There exists $t \in S^{\prime}$ so that $2 t \neq 0$.

Suppose $w \in S$. As in case $1, w \alpha$ is either $w$ or $-w$. If $w \alpha=w, w \in S^{\prime}$. Suppose then that $w \alpha=-w$. Since $\alpha$ is a congruence $w t=w \alpha t \alpha$ $=-w t\left(t \in S^{\prime}\right.$ and hence invariant under $\left.\alpha\right)$. Hence $w-t=w+t$ or $w-t=-w-t$. The first equation is impossible since $2 t \neq 0$. Therefore $w-t=-w-t$ and $w=-w=w \alpha$ so that $w \in S^{\prime}$. This shows that the identity mapping of $G$ (translation induced by 0 ) coincides with $\alpha$ over $S$.

The generalization of superposability theorems to non-abelian groups is not fruitful (although such groups have been studied from the metric viewpoint $)^{5}$ since in these studies it is shown that the reflections of such a group are motions if and only if the group is either abelian or is a Hamiltonian 2-group. ${ }^{6}$

UNIVERSITY OF MISSOURI

${ }^{5}$ Cf. O. Taussky, Math. Ann. vol. 108 (1933) pp. 615-620 and Quart. J. Math. Oxford Ser. vol. 12 (1941) pp. 64-67.

${ }^{6} \mathrm{~A}$ Hamiltonian 2-group is a group in which the order (period) of each element is a power of 2 . 